\title{
The carbon footprint of laparoscopic surgery: should we offset?
}

\author{
A. D. Gilliam · B. Davidson · J. Guest
}

Received: 16 October 2007/Accepted: 12 November 2007/Published online: 20 December 2007

(C) Springer Science+Business Media, LLC 2007

\begin{abstract}
The aim of this study was to estimate the effect that the expansion of laparoscopic surgery has had on global warming. Laparoscopic procedures performed in a hospital over a 10-year period were analysed. The number of $\mathrm{CO}_{2}$ cylinders (size $\mathrm{C}$ ) used over a 2.5 -year period and the "carbon footprint" of each cylinder was calculated. There was a fourfold increase of in the number of laparoscopic procedures performed over the past 10 years $(n=174-688)$. Median operative time for the laparoscopic procedures performed over the past 2.5 -years $(n=1629)$ was $1.01 \mathrm{~h}$ (range $0.3-4.45 \mathrm{~h}$ ) with 415 cylinders used in this period giving an operative time per cylinder of $3.96 \mathrm{~h}$. Each cylinder produces only 0.0009 of tonnes of $\mathrm{CO}_{2}$. Despite increasing frequency of the laparoscopic approach in general surgery, its impact on global warming is negligible.
\end{abstract}

Keywords Abdominal $\cdot$ Costs $\cdot$ Technical

Human-induced climate change through rapid exploitation of fossil fuels is the greatest social, economic, and environmental threat of this century. The principal "greenhouse" gas, carbon dioxide $\left(\mathrm{CO}_{2}\right)$, is used by

A. D. Gilliam $(\bowtie) \cdot$ J. Guest

Department of Surgery, Wansbeck Hospital, Newcastle,

Northumberland, UK

e-mail: andrewgilliam@btinternet.com

B. Davidson

Department of Engineering, Liverpool University, Liverpool, Merseyside, UK

A. D. Gilliam

Department of Surgery, North Tyneside Hospital, Newcastle,

Tyne and Wear, UK laparoscopic surgeons to establish pneumoperitoneum. This study aimed to estimate the effect that the expansion of laparoscopic surgery has had on global warming.

The annual number of laparoscopic procedures performed in a district general hospital over a 10 -year period (June 2006 to June 2007) was analyzed. The number of laparoscopic general surgery operations and the time required to perform all of them at the hospital were reviewed together with the number of $\mathrm{CO}_{2}$ cylinders (size C) used over a 2.5-year period (January 2005 to July 2007). The "carbon footprint" of each cylinder was calculated.

Over the past 10 years, the number of laparoscopic procedures performed has increased nearly fourfold, from 174 procedures to 688 procedures. The median operative time required for the laparoscopic procedures performed over the past 2.5 years $(n=1,629)$ was $1.01 \mathrm{~h}$ (range, 0.3 $4.45 \mathrm{~h}$ ). A total of 415 cylinders were used during this study period, providing a total operative time of $1,645 \mathrm{~h}$ (68.5 days) and giving an operative time of $3.96 \mathrm{~h}$ per cylinder. Each cylinder produces only 0.0009 tonnes of $\mathrm{CO}_{2}$, equivalent to a drive in a medium-sized petrol engine car of less than 1 mile. Because laparoscopic surgery uses only tiny amounts of $\mathrm{CO}_{2}$, its impact on global warming is minimal.

\section{Calculation}

Because 1 mole of any gas occupies 22.41 at 1 atmosphere and 1 mole of $\mathrm{CO}_{2}$ weighs $44 \mathrm{~g}$, then $44 \mathrm{~g}$ occupies $22.4 \mathrm{l}$, $4501=(44 / 22.4 \times 450) \quad \mathrm{g}, \quad 1 \mathrm{~kg}$ occupies $(1000 /$ 44) $\times 22.41,1$ tonne $[1,000 \mathrm{~kg}]$ occupies $1,000 \times] 1,000 /$ 44) $\times$ 22.4] $)=509,090.911$, and 1 tonne $=509,090.911$ or $11=1.96 \times 10-6$ tonnes. Therefore, 4501 is equivalent to $8.84 \times 10-4$ tonnes $=0.000884$ tonnes. 